\section{The Production of very Intense Magnetic Fields.}

IN a letter to NATURE which appeared in the issue for April I9 last, I gave a brief outline of a method for producing very intense magnetic fields.

I have now made some measurements which show that magnetic fields of considerably greater intensity may be generated by this method than had appeared at first sight to be practicable. For example, in one test a magnetising solenoid wound with 25.5 turns per $\mathrm{cm}$. was used, and the first peak value of the discharge current obtained when condensers of 700 micro-farads total capacity were used, charged to a pressure of I 850 volts, was found from the oscillograph record to be 12,500 amperes. This means that an intensity of magnetic field of about 400,000 gauss was generated.

By means of a modification of the apparatus which is now being effected, it is expected that an intensity of magnetic field of about I,500, ooo gauss will be obtained very shortly.

It is useful to examine what considerations govern

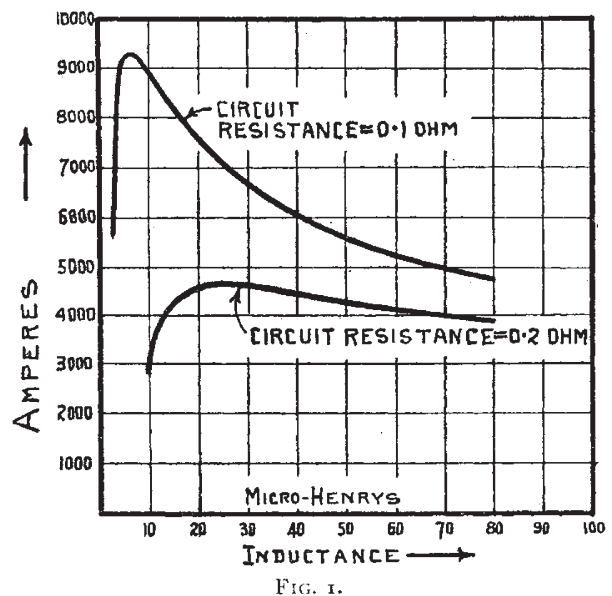

the maximum value of the condenser discharge current which can be obtained by this method. The magnitude of the first peak of the discharge current is given by the expression

$$
\frac{\mathrm{V}}{\mathrm{L} \sqrt{\frac{\mathrm{I}}{\mathrm{LC}}-\frac{\mathrm{I}}{4} \frac{\mathrm{R}^{2}}{\mathrm{~L}^{2}}}} e^{-\frac{\mathrm{r}}{2} \frac{\mathrm{R}}{\mathrm{L}} \mathrm{T}} \text { amperes, }
$$

where

$\mathrm{V}$ volts is the pressure to which the condenser is charged.

L henry is the, inductance of the circuit.

C farad is the total condenser capacity.

$\mathrm{R}$ ohm is the resistance of the circuit.

$\mathrm{T}$ second is the time for a quarter-period of the discharge current.

$e=2.718$ and is the base of natural logarithms.

In Fig. I the magnitude of the first peak of the discharge current is shown as a function of the inductance when the condenser capacity is 700 microfarads and the pressure 2000 volts. Two cases have been selected, namely, (i.) for a circuit resistance of $\mathrm{O} \cdot \mathrm{I}$ ohm, and (ii.) for a circuit resistance of $0.2 \mathrm{ohm}$.

It is important to observe that the maximum value of the first peak of the discharge current is obtained for a definite value of the inductance, and that this maximum value is more sharply defined the smaller the resistance of the circuit.

Edgar Allen Research Laboratory,

University of Sheffield,

$$
\text { August } 30 \text {. }
$$

\section{Absorption of Vapours of Various Liquids by Cotton.}

THE familiar absorption of water from the air by cotton has been studied in our laboratories for some years from various aspects. The phenomena are valuable as clues for interpreting the physico-chemical structure of cotton cellulose; and in this connexion we have naturally examined the capillary hypothesis, using Anderson's formula for calculation of capillary radii. We recently initiated a simple series of observations which indicate that this hypothesis is inadequate, by showing that the weight of vapour absorbed is dependent on the chemical nature of the vapour, with a suggestion that there is a constant " low level " group, including definite types of liquids, with which the values are due to simpler causes than with water or acetic acid, the values for the two latter being far higher. As the differences for various liquids are very marked and do not seem to have been previously recorded, the following particulars may be of interest, in spite of the primitive methods used in what was merely a preliminary examination.

Samples of unbleached and bleached twofold Egyptian cotton hosiery yarn were suspended, at room temperatures, in a bottle, in the bottom of which was a layer of the liquid under consideration. The cotton (which before enclosure had been dried for four hours in a water oven and then weighed) was withdrawn, with suitable precautions, at intervals of several hours and weighed in a weighing bottle. Curves were drawn through the plottings of these weight-values in order to show approximately the variation of weight with time for water, alcohol, acetic acid, benzene, nitrobenzene, carbon disulphide, ether and acetone. The following table shows only the saturation values

\begin{tabular}{|c|c|c|}
\hline Liquid. & Unbleached. & Bleached. \\
\hline & Per cent. & Per cent. \\
\hline Water $\cdot$ & 18 to 20 & I9 to $2 \mathrm{I}$ \\
\hline Glacial acetic acid & $18,, 20$ & I7 , I9 \\
\hline Absolute alcohol . & $3 \quad, \quad 3 \frac{1}{2}$ & $8 \frac{1}{2},, \quad 9$ \\
\hline Carbon disulphide & $x_{2}^{1}, 2$ & $\mathrm{x} \frac{1}{2}$, \\
\hline Benzene. & I $\frac{1}{2}$, & I ", \\
\hline Ether . & $7 "$ & 7, \\
\hline Nitrobenzene & $I \frac{1}{2}, \ldots 2$ & $I_{\frac{1}{2}}^{\frac{1}{2}}$, \\
\hline Acetone . & $2 \quad, \quad 2 \frac{1}{2}$ & $6 \frac{1}{2}$, \\
\hline
\end{tabular}
at room temperatures :

It is intended to investigate these phenomena further in the Experimental Department of the Fine Cotton Spinners' and Doublers' Association, to which I am indebted for permission to publish this note.

Rock Bank, Bollington, RoBERT C. Brimley.

near Macclesfield, Cheshire, August 29.

The Magnetic Resolution of the Scandium Lines.

SEVERAI lines of the scandium spectrum have been classified by Catalán (An. Soc. Esp., 20, 606, I922, and $2 I, 464,1923$ ). According to him, the Sc I. spectrum contains a doublet- and a quartet-term system; the Sc II. spectrum a triplet-system. It is, however, probable that some corrections must be made in his term-scheme. These corrections are obviously also made by Gieseler and Grotrian (Naturw., I 2,438 , I924).

We have made provisional measurements of the magnetic resolution of some scandium-lines. By means of the formulæe given by Landé (Zeit.f. Physi,

NO. 2864, VOL. I I 4 ] 